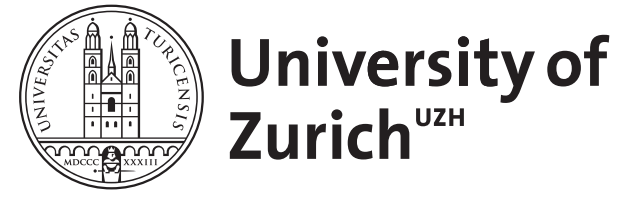

Zurich Open Repository and Archive

University of Zurich

University Library

Strickhofstrasse 39

CH-8057 Zurich

www.zora.uzh.ch

Year: 2006

\title{
Kognitive Verhaltenstherapie bei phobischer Angst vor dem Erbrechen
}

Rink, K

DOI: https://doi.org/10.1007/s00278-005-0468-4

Posted at the Zurich Open Repository and Archive, University of Zurich

ZORA URL: https://doi.org/10.5167/uzh-156155

Journal Article

Published Version

Originally published at:

Rink, K (2006). Kognitive Verhaltenstherapie bei phobischer Angst vor dem Erbrechen. Psychotherapeut, $51(3): 223-228$.

DOI: https://doi.org/10.1007/s00278-005-0468-4 
Psychotherapeut 2006 $\cdot$ 51:223-228 DOI 10.1007/s00278-005-0468-4

Online publiziert: 4. Januar 2006

(c) Springer Medizin Verlag 2006

Redaktion

U. Ehlert, Zürich

C. Reimer, Gießen

\section{Klaus Rink}

Abteilung Klinische Psychologie und Psychotherapie, Psychologisches Institut, Zürich

\section{Kognitive}

Verhaltenstherapie bei

phobischer Angst vor

dem Erbrechen
B ei den spezifischen Phobien findet man im Vergleich zu anderen Angststörungen - wie sie nach dem „Diagnostic and Statistical Manual of Mental Disorders(DSM-IV)“ oder der „International Classification of Diseases- (ICD-10)“ definiert und diagnostiziert werden - eine außerordentliche Vielfalt an Angstauslösern. Neben den häufig vertretenen Auslösern wie Spinnen, Schlangen, enge Räume oder Höhen gibt es, um nur einige Beispiele zu nennen, Personen, die auf Vogelfedern, tote Hasen, Gewitter oder auf Erbrechen mit phobischer Angst und ausgeprägtem Meideverhalten reagieren. In diesem Beitrag geht es um die Probleme und Besonderheiten bei der Therapie der Angst vor dem Erbrechen. Sie zählt zu den spezifischen Phobien, auch wenn gelegentlich der Begriff der „Emetophobie“ verwendet wird. Obwohl die Betroffenen häufig glauben, sie seien ein Unikat und viele Psychotherapeuten noch nie einen Patienten mit dieser Variante einer spezifischen Phobie in Behandlung hatten, handelt es sich keineswegs um eine seltene spezifische Phobie. Dies zeigt die Zahl der registrierten Mitglieder von Internet-Foren, in denen sich Betroffene über ihre Erfahrungen und Probleme austauschen. Im deutschen Forum http://www.emetophobie.de sind derzeit 705, im holländischen Forum http://www. emetofobie.nl sogar 1182 Mitglieder registriert worden. Den „geposteten“ Beiträgen der Mitglieder ist zu entnehmen, dass die überwiegende Mehrzahl von ihnen (noch) keine psychotherapeutische Hilfe gesucht bzw. in Anspruch genommen hat. Dasselbe gilt allerdings generell für spezifische Phobien. In vielen Fällen sind das durch die phobische Angst und das Meideverhalten verursachte Ausmaß an Lebenseinschränkung und der damit verbundene Leidensdruck nicht so hoch, dass therapeutische Hilfe gesucht wird. Andererseits wissen viele Betroffene nicht, wie man ihre Problematik Erfolg versprechend behandeln kann.

Bei der pathologischen Angst vor dem Erbrechen (Emetophobie) handelt es sich um eine unnatürliche, irrationale Angst gegenüber dem Erbrechen. Dabei können eine oder mehrere der folgenden Befürchtungen vorherrschend sein: Angst davor, miterleben zu müssen wie andere Personen und/oder Tiere sich übergeben, sich selbst zu übergeben und Angst vor jeglicher Konfrontation mit dem Thema „Erbrechen“ in Filmen, Printmedien oder Gesprächen. Die phobische Angst bei der
Konfrontation mit dem Erbrechen einer anderen Person unterscheidet sich deutlich vom „normalen“ Ekel vor Erbrochenem, den die meisten Menschen empfinden. Für die Diagnose einer spezifischen Phobie müssen die folgenden Kriterien gegeben sein:

- Die Angst ist stark ausgeprägt und besteht lange Zeit.

- Die Person ist sich bewusst, dass ihre Angst übertrieben ist (gilt nicht bei Kindern).

- Die phobischen Situationen werden gemieden oder nur unter Angst oder starkem Unbehagen ertragen.

- Die phobische Angst führt zu einer deutlichen Einschränkung der Lebensführung bzw. der beruflichen, schulischen oder sozialen Aktivitäten.

Wie bei allen spezifischen Phobien kann die Angst beim unmittelbaren Erleben des Erbrechens (einer anderen Person) panikartige Intensität mit Herzrasen, Beklemmungsgefühl, Schwindel, Unwirklichkeitsgefühl und Verwirrtheit erreichen. Bei starker Ausprägung der Phobie vor Erbrechen ist die Angst, zu erleben wie jemand anderes sich übergibt, un- 
terschwellig ständig vorhanden. Sie führt in der Regel zu ausgeprägtem Meideverhalten. Typischerweise werden öffentliche Orte und Veranstaltungen wie Jahrmärkte, Betriebsfeiern, Parties oder Discos gemieden, bei denen der Phobiker mit einem erhöhten Risiko rechnet, auf Betrunkene zu stoßen oder befürchtet, die Gesellschaft nicht frühzeitig verlassen zu können bevor Anwesende betrunken sind. Öffentliche Verkehrsmittel werden gemieden, sofern damit gerechnet wird, dass Betrunkene von einem Festanlass kommen oder dass Fahrgäste Reiseübelkeit bekommen und erbrechen könnten. Kinofilme, von denen man erfahren hat, dass eine Szene mit Erbrechen vorkommt, werden ebenfalls gemieden. Freunde und Bekannte werden oft präventiv befragt, ob in bestimmten Filmen solche Szenen vorkommen.

Noch schwerwiegender und ausgedehnter ist das Meideverhalten, wenn zusätzlich Angst vor dem eigenen Erbrechen besteht. Die Angst, sich zu überessen und erbrechen zu müssen, führt in vielen Fällen zu einer Sensibilisierung für Körperempfindungen (Völlegefühl und alle subtilen Formen von Unwohlsein) vor, während und nach der Nahrungsaufnahme, die als Vorläufer für wirkliche Übelkeit mit Erbrechen interpretiert werden. Das Essverhalten ist mitunter sehr restriktiv - wie bei einer Anorexie; grundsätzlich wird möglichst wenig und lange Zeit nichts gegessen. Daraus kann sich ein chronisches Untergewicht ergeben, das nicht selten zur Fehldiagnose einer Anorexie führt. In schweren Fällen kommt es zu einem ständigen „inneren“ Kontrollieren der Befindlichkeit, das gleich nach dem Wachwerden einsetzt und allenfalls durch sehr aufmerksamkeitsfordernde Tätigkeiten unterbrochen wird. Hat sich erst einmal eine leichte Form von Übelkeit bemerkbar gemacht, führt die stetige ängstlich-besorgte Aufmerksamkeit meistens dazu, dass das Unwohlsein bestehen bleibt oder sich noch steigert. Diese „Angst-Übelkeit“ kann von den Betroffenen oft nicht mehr von der Übelkeit vor einem Erbrechen unterschieden werden. Haltbarkeitsdaten aller Lebensmittel werden streng kontrolliert und Lebensmittel gemieden, von denen man fürchtet, dass sie Krankheitskeime enthalten könnten, die zu einer Erkrankung mit Übelkeit führen (Eier, Fisch, Fleisch). Werden im Krankheitsfall Medikamente verordnet, so wird deren Einnahme gemieden, sobald im Beipackzettel Übelkeit als mögliche Nebenwirkung verzeichnet ist. In vielen Fällen verlassen die Betroffenen nur ungern - und wenn, nur für kurze Zeit die eigene Wohnung, weil sie außerhalb der Wohnung nichts essen und das Risiko eigenen Erbrechens minimieren wollen. Von Fremden zubereitete Speisen werden hinsichtlich ihrer Frische und Keimfreiheit als zu gefährlich betrachtet. Sobald der Betroffene Informationen über grassierende Viren bekommt oder diese aufgrund von Jahreszeit und Wetter erwartet, wird der Aufenthalt außerhalb der eigenen Wohnung bzw. die Gesellschaft vieler Menschen ebenfalls gemieden. In fremden Räumen wird zwanghaft nach Fluchtwegen, Mülleimern oder Waschbecken gesucht, in die man sich im Notfall übergeben kann. Die genannten Einschränkungen machen in vielen Fällen den kontinuierlichen Besuch einer Schule, das Absolvieren einer Ausbildung oder eines Studiums unmöglich. Selbst der Besuch von Freunden oder Angehörigen wird eingeschränkt oder vermieden, wenn sich dort Schwangere oder Kleinkinder aufhalten oder wenn jemand erkrankt ist, weil dann das subjektive Risiko, ein Erbrechen miterleben zu müssen, steigt. Manche Patienten nehmen Antibrechmittel, wenn sie Übelkeit verspüren, um tatsächliches Erbrechen zu verhindern. In solchen Fällen kann es zu einem exzessiven Missbrauch dieser Medikamente kommen.

Es soll darauf hingewiesen werden, dass diese Auflistung summarisch über viele Personen mit pathologischer Angst vor dem Erbrechen entstanden ist. Sie bietet einen Anhaltspunkt zum Erfragen des individuell gegebenen Meideverhaltens und zur Einschätzung des Schweregrades der Störung.

Bei spezifischen Phobien sind - wie bei anderen Angststörungen - Varianten der verhaltenstherapeutischen Reizkonfrontation (Exposition mit den Angst auslösenden Objekten oder Situationen) und dem damit verbundenen systematischen Abbau des Meideverhaltens erwiesenermaßen die wirksamsten Behandlungsmethoden. In kontrollierten Vergleichsgrup- penstudien liegen die Erfolgsquoten (klinisch signifikanten Verbesserungen) für die jeweils beste Therapievariante zwischen 77 und 95\% (Öst 1996). Trotz dieser sehr günstigen Voraussetzungen kann die angesprochene Vielfalt an Auslösern zu Behandlungsproblemen führen. Bei manchen Auslösern handelt es sich um Ereignisse, die nicht ohne weiteres herstellbar sind und deren Eintreten zeitlich oder örtlich nur sehr schwer oder gar nicht zu bestimmen ist (beispielsweise Gewitter, Sturm, Erbrechen anderer Personen). Für den Erfolg einer Reizkonfrontationstherapie in vivo ist aber gerade die geplante Herstellung der Auslöser in ausreichender Intensität, Dauer und Häufigkeit äußerst wichtig.

Eine zweite Schwierigkeit für die erfolgreiche Therapie einer spezifischen Phobie ist dann gegeben, wenn die Intensität bzw. Ausweitung des Meideverhaltens mit positiven sekundären Folgen verbunden sind (gesteigerte soziale Zuwendung, Verringerung unangenehmer Verpflichtungen, Durchsetzung eigener Ansprüche), die zu einem Zielkonflikt (Motivationsproblemen) führen. Im Folgenden werden zwei Fallvignetten mit phobischer Angst vor dem Erbrechen vorgestellt, bei denen eines oder mehrere der oben genannten Probleme gegeben waren.

\section{Fall 1}

\section{Situation}

Die 32-jährige Patientin hatte seit vielen Jahren eine ausgeprägte Phobie vor Situationen, in denen eine andere Person erbrechen muss. Die Entwicklung der Phobie begann, als die damals 6-jährige Patientin zu Hause für einige Stunden auf ihre 3-jährige Schwester aufpassen sollte. Sie spielte mit ihrer Schwester auf dem Teppich im Wohnzimmer, als diese plötzlich erbrechen musste. Die Patientin erschrak sehr und war entsetzt, weil der Wohnzimmerteppich und ihre Kleidung mit Erbrochenem verschmutzt waren. Sie weinte und war noch für längere Zeit untröstlich, als ihre Eltern wenig später kamen und sie zu beruhigen versuchten. Die jüngere Schwester neigte zu Übelkeit beim Autofahren. Bei Fahrten mit der Familie kam es wiederholt zu Situationen, in denen die 
Schwester äußerte, dass ihr schlecht sei. Obwohl die Fahrt jedes Mal rechtzeitig unterbrochen wurde und es nicht zum Erbrechen kam, reagierte die Patienten mit heftiger Angst und Weinen, als die Möglichkeit bestand, dass ihre Schwester erbrechen könnte. Über die Jahre hinweg erlebte die Patientin wiederholt Situationen, in denen jemand erbrach, obwohl sie inzwischen viele Situationen mied, in denen damit gerechnet werden konnte. Wenn ein Stadtfest veranstaltet wurde, mied sie nicht nur in die Nähe des Festgeländes zu gehen, sondern auch die Nutzung der Verkehrsmittel, mit denen Festgäste vom Fest zurückfuhren. Wenn sie erfuhr, dass im Freundes- und Bekanntenkreis jemand erkrankt war oder vor kurzem gar erbrechen musste, mied sie eine beträchtliche Zeit lang alle Gelegenheiten, mit diesen Personen in Kontakt zu kommen. Wenn ihr Lebensgefährte abends mit Freunden ausging und dabei Alkohol getrunken wurde, fürchtete sie, dass ihm schlecht werden könnte, wenn er spät abends wieder nach Hause kam. Sie konnte an solchen Tagen nicht mit ihm in einem Raum schlafen. Dass sie nach vielen Jahren seit Bestehen dieser Phobie erstmals therapeutische Hilfe suchte, hatte zwei Gründe. Ihr Lebenspartner war engagiertes Mitglied im lokalen Karnevalsverein. Zum Vereinsleben gehörten viele Veranstaltungen mit einer großen Besucherzahl, bei denen traditionell viel getrunken wurde, und bei denen es sehr häufig dazu kam, dass jemand, der „über den Durst getrunken“ hatte, erbrechen musste. Sie wollte sich nicht dauerhaft von den Aktivitäten ihres Partners fernhalten, weil auch ein großer Teil ihres gemeinsamen Freundeskreises daran beteiligt war. Außerdem planten die beiden, zu heiraten und Kinder zu bekommen. Die Patientin hatte große Sorge, dass sie ihr Kind in einer Panik fallen lassen könnte, wenn dieses erbrechen würde, während sie es trägt.

\section{Behandlungsplan und Behandlungsproblem}

Es gab zwei klare Behandlungsziele. Zum Einen sollte die intensive, manchmal panikartige Reaktion auf das unverhoffte Miterleben des Erbrechens einer anderen Person durch ein Habituationstraining mit Angst auslösenden Reizen abgebaut werden, sodass die Patientin künftig alle möglichen Situationen, in denen jemand erbricht, angstfrei ertragen kann, und dass sie ihr Kind problemlos halten, versorgen sowie beschmutzte Wäsche reinigen kann, wenn es erbrechen muss. Zum Anderen sollte das Meideverhalten abgebaut werden. Die Patientin sollte gezielt alle Situationen, die sie in der Vergangenheit aus Furcht, es könne jemand erbrechen, gemieden hat, aufsuchen. Sie sollte also Freunde besuchen, obwohl diese erkrankt sind und gemeinsam mit dem Partner in hoher zeitlicher Dichte Feste besuchen und öffentliche Verkehrsmittel benutzen, in denen Festgäste von großen Festanlässen kommen. Dem systematischen Aufsuchen gemiedener Situationen sollte dabei eine erfolgreiche Habituationserfahrung bei therapeutischen Reizkonfrontationen vorausgehen. Das Behandlungsproblem bestand darin, für die Reizkonfrontation fremdes Erbrechen „herzustellen“ und so lange andauernd oder wiederholt darzubieten, bis die anfänglich intensive Angst der Patientin stabil habituieren (auf ein geringes Niveau sinken) konnte. Der Besuch von öffentlichen Festveranstaltungen hätte eine Konfrontation mit dem gefürchteten $\mathrm{Er}$ eignis zwar durchaus wahrscheinlich gemacht, aber keineswegs sichergestellt. Hinzu kommt, dass es bei großen Veranstaltungen eher zufällig wäre, ob die $\mathrm{Pa}$ tientin sich gerade in der Nähe befindet und den Vorfall miterleben kann. Das Aufsuchen solcher Veranstaltungen fordert einen großen Zeitaufwand, ohne sicherzustellen, dass die gewünschte Konfrontation mit dem Ereignis stattfindet. Hinzu kommt, dass die Dauer des einmaligen Ereignisses viel zu kurz ist, um eine „Erschöpfung“ der Angst (Habituation) sicherzustellen. Es ist nahe liegend, Filmszenen, in denen Erbrechen gezeigt wird, zur Reizkonfrontation zu verwenden. Sofern ein Film mit einer Szene gefunden ist, der bei der Patientin in ausreichendem Maße Angst auslöst, kann man diese Szene so oft wiederholt ansehen lassen, bis Angst, Unwohlsein und Meidenwollen dauerhaft nachgelassen haben und die Patientin das sichere Gefühl hat, sie könnte die Szene noch beliebig oft ansehen, ohne dass die Angst wiederkommt. Bei Verwendung einer einzigen Szene wäre allerdings zweifel- haft, ob sich die Angstreduktion auf unterschiedliche reale Situationen übertragen würde. Um die Wahrscheinlichkeit hierfür zu steigern, wurden Szenen mit Erbrechen aus 17 verschiedenen Filmen mit einer Dauer von 21 Minuten zusammengeschnitten, viermal hintereinander kopiert und auf eine DVD gebrannt. Damit stand für die Reizkonfrontation ein Film von insgesamt 84 Minuten mit Erbrechen zur Verfügung.

\section{Behandlungsverlauf}

Die Patientin wurde über das Rationale und die Wirkung einer verhaltenstherapeutischen Reizkonfrontation bei Ängsten ausgiebig informiert, und der Behandlungsplan wurde mit ihr besprochen. Die erste Reizkonfrontation (das wiederholte Ansehen der Erbrechensszenen) fand in Anwesenheit des Therapeuten statt. Der erste Durchgang mit allen Szenen löste heftige Angst und Unwohlsein aus. Nach einer Konfrontationsdauer von ca. 2 Stunden und 15 Minuten war der erwünschte Grad an Habituation erreicht, der auch beim weiteren Betrachten erhalten blieb. In der folgenden Woche führte die Patientin zu Hause tägliche Reizkonfrontationen mit der DVD durch. Nach wenigen Tagen wurde bei einer Konfrontationsdauer von 30 Minuten eine anhaltende Habituation erreicht. In der dritten Woche wurde die Häufigkeit der Reizkonfrontation auf dreimal wöchentlich reduziert. Stattdessen kamen inhaltlich und terminlich festgelegte Übungen zum Abbau des Meideverhaltens hinzu. Dieses kombinierte Vorgehen wurde über einen Zeitraum von 6 Wochen weitergeführt, bis die Patientin beim Betrachten der Erbrechensszenen und beim Aufsuchen bisher gemiedener sozialer Situationen weder Angst noch Unbehagen oder den Drang zum Verlassen der Situation verspürte.

\section{Fall 2}

\section{Situatation}

Beim ersten Fall bestand das Behandlungsproblem in der Herstellung des Materials für die systematische Reizkonfrontation. Die Behandlung selbst verlief problemlos. Beim 2. Fall konnte auf das be- 
reits erstellte Videoband zur Reizkonfrontation zurückgegriffen werden. Es ergaben sich aber beim Behandlungsversuch mehrere Probleme, die schließlich zur Unterbrechung und Sistierung der Behandlung führten. Die 27-jährige Patientin hatte nicht nur Angst, dass andere in ihrer Anwesenheit erbrechen könnten, sondern sie hatte auch Angst, selbst zu erbrechen. Sie hatte ein äußerst restriktives Essverhalten und ein ausgeprägtes (agoraphobisches) Meideverhalten. Zum Zeitpunkt des Behandlungsbeginns war sie stark untergewichtig (44 kg, Bodymass-Index 16). Die Entwicklung der phobischen Angst vor dem fremden und eigenen Erbrechen begann mit einem Ereignis in der Grundschulzeit der Patientin. Die Tischnachbarin der damals 7-jährigen Patientin musste völlig überraschend mitten im Unterricht erbrechen, sodass der gemeinsame Schultisch, Schulhefte, Bücher und Teile der Bekleidung der Patientin verschmutzt waren. Die Patientin reagierte mit entsetztem Schreien und anhaltendem Weinen. Die Lehrerin musste sich so sehr mit ihr beschäftigen und hatte größte Mühe, sie irgendwie zu beruhigen, dass ihr kaum Zeit blieb, sich um das Mädchen zu kümmern, dem schlecht geworden war. In der Folgezeit ist es wohl zu einer Sensibilisierung für Situationen gekommen, in denen die Tischnachbarin, andere Schüler und schließlich auch Personen außerhalb der Klasse erbrechen könnten. Trotzdem kam es noch nicht zu einem sich generalisierenden Meideverhalten. Lehrerin und Eltern wirkten offenbar so auf die Schülerin (die Patientin) ein, dass sie weiterhin regelmäßig die Schule besuchte. $\mathrm{Zu}$ einer erheblichen Steigerung der Sensibilität und des phobischen Verhaltens kam es erst, als sich die Patientin ca. 3 Jahre vor dem Abitur, bedingt durch große Lernschwierigkeiten (schlechte Zensuren und Zerwürfnisse mit Lehrern) in einer sehr krisenhaften Lebenssituation befand. In dieser Zeit entwickelte sie zusätzlich zur Furcht vor fremdem Erbrechen, die Angst vor eigenem Erbrechen sowie eine Sensibilisierung für körperliche Empfindungen des Unwohlseins und das restriktive Essverhalten. Einige Monate später, etwa 2 Jahre vor dem Abitur brach die Patientin die schulische Laufbahn ab, nachdem verschiedene Bemühungen seitens der
Lehrer und der Eltern gescheitert waren, die Lern- und Motivationsprobleme sowie das zunehmende (emeto-)phobische Meideverhalten der Patientin, das immer häufiger zu Absenzen in der Schule führte, $\mathrm{zu}$ reduzieren. Aus verhaltenstherapeutischer Sicht ist, gestützt auf die Aussagen der Patientin, zu vermuten, dass die chronischen Schulschwierigkeiten eine physiologische Erregung verursachten, die u. a. auch zu Unwohlsein und latenter Übelkeit geführt haben. Die damit „entschuldigten“ schulischen Absenzen führten nicht nur zu einer Reduktion der Angst, fremdes oder eigenes Erbrechen in der außerhäuslichen Öffentlichkeit zu erleben, sondern auch zu einer Reduktion des Drucks, sich den eigenen schulischen Schwierigkeiten stellen zu müssen. Das Fernbleiben wurde also zweifach negativ bekräftigt. Retrospektiv schwer zu klären ist dabei, inwieweit die Steigerung der emetophobischen Symptome in der Kommunikation mit Eltern und Lehrern auch intentional eine gute Rechtfertigung für das Fernbleiben von der Schule war. Im genannten 2Jahres-Zeitraum nahm die Patientin erstmals eine 20-monatige, psychotherapeutische Behandlung in Anspruch, mit der die spezifische Phobie vor dem Erbrechen jedoch nicht nachhaltig reduziert werden konnte. Sie lebte nach dem Schulabbruch mehrere Jahre im Elternhaus, ohne eine schulische oder berufliche Ausbildung aufzunehmen. Das Verhältnis zum Vater war durch anhaltende verbale Auseinandersetzungen geprägt, in denen es der $\mathrm{Pa}$ tientin nach eigenen und nach Aussagen der Mutter fast immer gelang, den Vater durch schier „endlose“ Diskussionen so $\mathrm{zu}$ zermürben, dass er aufgab und die $\mathrm{Pa}$ tientin das Gefühl bekam, immer Recht behalten zu haben. Der ersten Psychotherapie folgten noch eine 3 -jährige und eine 8-monatige, die ebenso wenig zu einer Reduktion des Meideverhaltens und des gestörten Essverhaltens führten. Nach einer 4-jährigen Therapiepause suchte die Patientin - vorinformiert durch die deutschsprachige Webseite zur "Emetophobie“ erstmals nach einer verhaltenstherapeutischen Behandlung. Sie nannte drei Therapieziele: die Normalisierung ihres Essverhaltens verbunden mit einer Gewichtszunahme, die Verringerung ihres Meideverhaltens, das ihr die Wiederaufnahme und reguläre Weiterführung ihres kurz zuvor begonnenen Fachhochschulstudiums ermöglichen sollte, und die Verringerung der Angst begleitenden und Angst verstärkenden körperlichen Symptome. Zur Erreichung dieser Ziele wurden wiederholte Reizkonfrontationssitzungen mit dem Zusammenschnitt von Erbrechensszenen und ein gestuftes Programm zum Abbau des Meideverhaltens geplant. Die Patientin sollte zunehmend längere Fahrten mit ihrem Motorroller und weitere Exkursionen mit dem Zug und zu Fuss unternehmen. Sie sollte dabei ihren Aktionsradius zunehmend erweitern und jedes Mal fest vereinbarte Mengen essen, damit sie die wiederholte Erfahrung macht, dass sie nicht erbrechen muss, obwohl sie regelmäßig außerhalb der Wohnung Nahrung zu sich nimmt. Die Vigilanz für körperliche Missempfindungen sollte durch Selbstinstruktionen zur „Gelassenheit“, durch aufmerksamkeitsfordernde Aktivitäten und durch aufklärende und einstellungsändernde Gespräche zur Entstehung und Aufrechterhaltung Angst bezogener Körperempfindungen verringert werden.

\section{Behandlungsverlauf und Behandlungsprobleme}

Obwohl die Patientin regelmäßig zu den therapeutischen Sitzungen kam und sehr gut über das kognitiv-verhaltenstherapeutische Rationale informiert war, zeigte sie bei allen oben genannten therapeutischen Maßnahmen eine ambivalente Motivation, wenn es um die konkrete Umsetzung ging. Trotz ausgiebiger Planung und Vorbesprechung wollte sie die erste gemeinsam mit dem Therapeuten durchgeführte Konfrontation mit den filmischen Erbrechensszenen bereits nach der ersten Szene abbrechen. Sie ließ sich erst nach einer sehr langen Diskussion zur weiteren Reizkonfrontation überreden, bis für die erste Szene eine vollständige Habituation erreicht werden konnte. Die terminlich und inhaltlich genau verabredeten selbstständigen Konfrontationsübungen führte sie größtenteils nicht oder nur unvollständig durch. Ein ähnliches Verhalten zeigte sie hinsichtlich der vereinbarten Exkursionen zum Abbau des Meideverhaltens und des Essens außerhalb der Wohnung. Dies machte gemeinsame Gespräche zur 
gründlichen Motivationsanalyse erforderlich. Dabei konnten zwei wesentliche, nicht voneinander unabhängige Hindernisse für das konsequente Umsetzen der Übungen identifiziert werden. Die Patientin hatte eine Hypervigilanz für körperliche Empfindungen, die mit einer ständig vorhandenen ängstlich-besorgten Grundhaltung verbunden war, dass sie erste Anzeichen für eine Übelkeit wahrnehmen könnte. Oft hatte sie schon morgens kurz nach dem Aufwachen beunruhigende Empfindungen (leichte Bauchschmerzen, Blähungen, Kloßgefühl im Hals). Die Patientin hatte die Einstellung, dass Konfrontationsübungen dann für sie unzumutbar sind, wenn solche Missempfindungen vorhanden sind, und wenn sie an diesem Tag noch etwas außer Haus erledigen musste und sich dadurch belastet fühlte. Sie empfand die Übungen dann als eine Zusatzbelastung, die die subjektive Grenze des Zumutbaren überschritt. Anders formuliert, sie war nur an den Tagen zu Übungen bereit, an denen sie sich ohne beunruhigende körperliche Beschwerden fühlte. Das zweite Hindernis war noch grundsätzlicher. Die Patientin hatte implizit einen völlig anderen Zeithorizont für die Erreichung ihrer Therapieziele als derjenige, der in den vorbereitenden Gesprächen als Orientierung explizit besprochen wurde. Die Patientin äußerte „Ich kann doch unmöglich meine Angst in wenigen Wochen aufgeben, wenn ich über viele Jahre hinweg mein ganzes Leben und meine Beziehungen so arrangiert habe, dass ich mit der Angst leben kann. Ich müsste mir ja sagen, das war alles völlig verrückt und sinnlos." Auf die Frage, wie viel Zeit sie sich für das angstfrei werden, nehmen möchte, sagte sie „zwei bis drei Jahre“. Die Patientin hatte die Wahl ihrer freundschaftlichen Beziehungen und ihres Lebenspartners sehr stark daran orientiert, ob diese viel Verständnis, Entgegenkommen und Unterstützung für ihre Angst aufbringen. Sie hatte also einen sekundären Krankheitsgewinn. Die von Freunden und dem Partner angebotene Hilfe, die erbrachten Opfer und Zugeständnisse sind gewissermaßen Beweise für den Wert und die Wichtigkeit der eigenen Person. Hinzu kommt, dass die Patientin selbst ihrer Angst enorm viel geopfert hat. Sie hat den Besuch des Gymnasiums und das Anstre- ben des Abiturs aufgegeben und sich erst mit einer mehrjährigen Verzögerung um die Zulassungsprüfung und Aufnahme in das Fachhochschulstudium bemüht. Aus der Interaktion der Patientin ließ sich ein weiteres motivationales Hindernis für die konsequente Verfolgung der Therapieziele erschließen. Die Patientin bemühte sich einerseits um eine freundliche, akzeptierende und wertschätzende Haltung in der therapeutischen Interaktion, sie machte aber immer wieder entwertende Aussagen über die Kompetenz der früheren Therapeuten, der Ärzte und teilweise auch über den verhaltenstherapeutischen Ansatz zur Erklärung und Behandlung ihrer Angst. Sie selbst räumte diesbezüglich ein, dass sie schon immer ein sehr kompetitives Verhältnis zu „Autoritäten“ und übergeordneten Personen hatte, und dass sie mit diesen immer wieder heftige Diskussionen hatte, bei denen sie Recht behalten wollte.

\section{Konsequenzen für die weitere Behandlung/Lösung des Problems}

$\mathrm{Zu}$ allererst sollte der Therapeut Akzeptanz und volles Verständnis dafür zeigen, dass es der Patientin schwer fällt, sich nach so vielen Jahren der angsterfüllten Beschäftigung mit dem Erbrechen und der starken Einschränkung ihrer Aktivitäten auf eine schnelle Änderung ihres Lebens einzustellen. Weiterhin ist es wichtig, darauf hinzuweisen, dass eine verhaltenstherapeutische Behandlung schwerer Ängste ein sehr hohes Maß an Motivation voraussetzt, weil der Patient schließlich genau das tun soll, was er jahrelang ausschließlich gemieden hat, nämlich sich wiederholt aktiv mit stark Angst auslösenden Reizen zu konfrontieren sowie dauerhaft gemiedene Situationen gezielt und wiederholt aufzusuchen. Aus diesem Grund wurden drei Sitzungen ausschließlich dazu verwendet, gemeinsam mit der Patientin nach allen motivationalen Hindernissen (Einstellungen, Überzeugungen, Gewohnheiten, Gefühle, Veränderungsperspektive usw.), die einer erfolgreichen Therapie entgegenstehen könnten, zu suchen. Nach einer solchen ausgiebigen Motivationsanalyse müssen Patientin und Therapeut gemeinsam entscheiden, ob und wie die begonnene Therapie weitergeführt werden soll. Entscheidend ist hierfür, ob es der Patientin gelungen ist, sich ihrer ambivalenten Gefühle, Einstellungen und Schlussfolgerungen bewusst zu werden und sie $\mathrm{zu}$ einer verbindlichen Übernahme und Verfolgung ihrer Therapieziele bereit ist. Ist dies der Fall, sollte man die Patientin auffordern, jeweils selbstständig die Wochenpläne mit den hinsichtlich Ort, Zeit, Inhalt und Dauer konkret festgelegten Konfrontations- und Exkursionsübungen zu gestalten. Die selbst gestalteten Wochenpläne geben wertvollen Aufschluss darüber, wie motiviert die Patientin ist (Marks 1993). Sofern die Ambivalenz weiter besteht und eine verbindliche Verfolgung der Therapieziele in einem vom Therapeut und dem Patienten gemeinsam gut geheißenen Zeitrahmen nicht möglich scheint, so ist es m. E. sinnvoll, die Therapie so lange auszusetzen, bis der Patient die Ambivalenz auflösen konnte und motiviert ist, die Übungen zur Konfrontation und zum Abbau des Meideverhaltens in einer therapeutisch wirksamen Intensität und zeitlichen Dichte durchzuführen. Im vorliegenden Fall wurde so verfahren. Die Patientin fühlte sich nach der Motivationsanalyse noch nicht für eine konsequente Weiterführung der Therapie bereit. Sie versprach sich wieder zu melden, sobald dies der Fall ist.

\section{Fazit für die Praxis}

Die Angst vor dem Erbrechen ist eine überraschend weit verbreitete Variante einer spezifischen Phobie. Die Konfrontation mit natürlich vorkommendem Erbrechen ist nicht so herstellbar, dass damit eine Erfolg versprechende kognitiv-verhaltenstherapeutische Behandlung durchgeführt werden könnte. Ein Zusammenschnitt filmischer Szenen des Erbrechens ermöglicht die therapeutisch begleitete und die selbstständige Reizkonfrontation des Patienten in beliebigem zeitlichen Umfang und beliebiger Häufigkeit. Schwerwiegendere Behandlungsprobleme ergeben sich, wenn der Patient einen mehr oder weniger bewussten Zielkonflikt hat, indem er sekundären Nutzen aus den Folgen seiner spezifischen Phobie ziehen kann (Unterstützung und Zuwendung durch andere, Entlastung/Vermeidung von unangenehmen Pflichten und Situationen) und 
wenn ein, therapeutisch gesehen, dysfunktionaler Zeithorizont für die gewünschte therapeutische Änderung besteht. In solchen Fällen ist eine ausgiebige Motivationsanalyse erforderlich, die entweder in eine Auflösung der Ambivalenz und Weiterführung der Therapie oder eine Unterbrechung und Sistierung der Therapie münden kann.

\section{Korrespondierender Autor PD Dr. phil. Klaus Rink}

Abteilung Klinische Psychologie und Psychotherapie, Psychologisches Institut, Universität, Zürichbergstrasse 43,

$\mathrm{CH}-8044$ Zürich

E-Mail:k.rink@psychologie.unizh.ch

Interessenkonflikt: Es besteht kein Interessenkonflikt. Der korrespondierende Autor versichert, dass keine Verbindungen mit einer Firma, deren Produkt in dem Artikel genannt ist, oder einer Firma, die ein Konkurrenzprodukt vertreibt, bestehen. Die Präsentation des Themas ist unabhängig und die Darstellung der Inhalte produktneutral.

\section{Literatur}

Marks I (1993) Ängste - Verstehen und bewältigen. Springer, Berlin Heidelberg New York Tokyo

Öst LG (1996) Spezifische Phobien. In: Margraf J (Hrsg) Lehrbuch der Verhaltenstherapie, Bd 2. Springer,

Berlin Heidelberg New York Tokyo

\section{Warum Borderline-Patientinnen weniger Schmerzen spüren}

Ein Forscherteam aus Mannheim und Mainz wurde mit dem diesjährigen Förderpreis für Schmerzforschung ausgezeichnet.

Der Preis wurde für die Erforschung eines völlig neuartigen, aktiven neurobiologischen Mechanismus der Schmerzunterdrückung durch zentralnervöse Plastizität vergeben.

Entdeckt wurde dieser Antinozizeptions-Mechanismus im Rahmen einer Studie, deren Ziel es war, den sog. hypoalgetischen Zustand zu charakterisieren. In diesem Zustand nehmen Patienten, die sich im Rahmen einer Borderline-Persönlichkeitsstörung (BPS) selbst verletzen, wenig oder überhaupt keinen Schmerz wahr.

Im Versuch setzten die Forscher BPS-

Patientinnen und gesunde weibliche Kontrollpersonen kurzen Hitzereizen aus. Die Patientinnen wiesen höhere Wahrnehmungs- und Schmerzschwellen für Laserreize auf und gaben eine verringerte subjektive Schmerzhaftigkeit an. Währenddessen wurden mittels EEG Laser-evozierte Hirnpotenziale (LEPs) abgeleitet, um die Verarbeitung der schmerzhaften Reize im Gehirn zu dokumentieren. Die Größen der unterschiedlichen LEP-Komponenten waren - als objektive Parameter - unverändert oder sogar leicht vergrößert. Die spätere LEP-Komponente P3, ein Maß für die Aufmerksamkeit, unterschied sich zwischen den beiden Gruppen nicht. Auch war die räumliche Diskriminationsleistung nicht von der Gesunder verschieden.

Somit bestätigt diese Studie frühere Befunde einer reduzierten Schmerzwahrnehmung bei Patienten mit BPS. Eine generelle Beeinträchtigung der sensorischdiskriminativen Schmerzverarbeitung konnte jedoch erstmals vollständig ausgeschlossen werden. Aus dieser Beobachtung folgerten die Forscher, dass das periphere System der Schmerzwahrnehmung sowie die frühe Verarbeitung schmerzhafter Reize im Gehirn bei Patientinnen mit BPS vollständig intakt sein müssen.

Diese Befunde sind einerseits für das Verstehen der Borderlinestörung von
Bedeutung, andererseits erhoffen sich die Forscher vom genaueren Verständnis dieses Phänomens auch eine Verbesserung in der Therapie chronisch gesteigerter Schmerzen.

\section{Quelle: Zentralinstitut für Seelische} Gesundheit, Mannheim 